\title{
Mifepristone Utilization in Cushing's From Large Bilateral Adrenal Adenoma Prior to Surgery
}

\author{
Vasudev Magaji ${ }^{\mathrm{a}, \mathrm{d}}$, Sarah Park $^{\mathrm{b}}$, Katie Mastoris ${ }^{\mathrm{b}}$, Maura Bucciarelli ${ }^{\mathrm{b}}$, Scott Beman ${ }^{\mathrm{c}}$
}

\begin{abstract}
Utilization of mifepristone was described to mitigate hypercortisolism from bilateral adrenal Cushing's syndrome. A 47-year-old female on abdominal pain evaluation with CT was found to have a $5.4 \times 1.7 \times 4.9 \mathrm{~cm}$ right adrenal nodule and a $4.3 \times 2.9 \times 3.3 \mathrm{~cm}$ left adrenal nodule with calcification on $\mathrm{CT}$ scan with each having density of -3 Housefield units. She had hypercortisolism with testing revealing high urine free cortisol, unsuppressed cortisol on $1 \mathrm{mg}$ and $8 \mathrm{mg}$ overnight dexamethasone suppression testing, high $11 \mathrm{pm}$ salivary cortisol $\times 2$ along with elevated random cortisol and undetectable ACTH. Her DHEA-S, plasma metanephrine, and rostenedione, renin and aldosterone levels were normal. Adrenal venous sampling for lateralization of hypercortisolism source using dexamethasone 2 mg PO Q 6 hours $\times 1$ day, showed adrenal vein epinephrine levels step up $>100 \mathrm{pg} / \mathrm{mL}$, indicating successful catheterization. Each side adrenal vein to vena caval cortisol ratio $>4$ was consistent with autonomous cortisol secretion and left to right adrenal cortisol ratio < 2 suggested bilateral adrenal Cushing's syndrome. She had multiple co-morbidities including uncontrolled hypertension, poor functional status requiring walker and cane, chronic non-healing cellulitis, glucose intolerance and hypokalemia. Mifepristone was initiated for medical management of hypercortisolism prior to surgery, resulting in $>50 \mathrm{lbs}$ weight loss, improved blood pressure, complete healing of chronic lower extremity cellulitis and independent ambulation within 4 months. Initially right adrenal adenoma and then left adrenal adenoma were resected in two separate surgeries and pathology showed bilateral enlarged adrenal glands with multiple adrenocortical nodules without adrenal carcinoma. Mifepristone, FDA approved for inoperable Cushing's, facilitated pre-surgery optimization by effectively mitigating hypercortisolism. Mifepristone was utilized as a bridge for
\end{abstract}

Manuscript accepted for publication June 24, 2015

aDivision of Endocrinology, Department of Medicine, Lehigh Valley Health Network, Allentown, PA 18103, USA

${ }^{b}$ Department of Medicine, Lehigh Valley Health Network, Allentown, PA 18103, USA

'Department of Surgery, Lehigh Valley Health Network, Allentown, PA 18103, USA

${ }^{\mathrm{d} C}$ Corresponding Author: Vasudev G. Magaji, Division of Endocrinology, Department of Medicine, Lehigh Valley Health Network, Allentown, PA 18103, USA. Email: vasudevgmagaji@gmail.com

doi: http://dx.doi.org/10.14740/jem288w
Cushing's medical management prior to surgery.

Keywords: Mifepristone; Cushing’s syndrome; Bilateral adrenal adenoma

\section{Introduction}

Cushing's syndrome characterized by glucocorticoid excess causes weight gain, hypertension, muscular weakness, glucose metabolism imbalance, proximal muscle weakness, poor tissue healing and increased susceptibility to infection. Cushing's syndrome not only presents challenges in identifying the cause of glucocorticoid excess but also presents unique management challenges. We present a case detailing diagnostic challenges of Cushing's syndrome resulting from bilateral ACTH-independent macronodular adrenal hyperplasia, unique and novel management strategy of using mifepristone to mitigate hypercortisolism and its effects including weight, hypertension, secondary infection for preoperative management of clinical manifestations of Cushing's syndrome. This not only helped in preparing the patient for surgery, addressed the increased risk factors for the surgery but also potentially expedited postoperative recovery by avoiding effects of abrupt glucocorticoid withdrawal following surgery.

\section{Case Report}

A 47-year-old female with past medical history significant for hypertension, hyperlipidemia, glucose intolerance, carotid artery disease, peripheral vascular disease, coronary artery bypass grafting, and chronic leg cellulitis presented with abdominal pain. Her CT scan of abdomen showed right adrenal nodule $1.8 \times 4.9 \mathrm{~cm}$ and left adrenal nodule $2.8 \times 4.3 \mathrm{~cm}$ (Fig. 1). She had workup for pheochromocytoma and hyperaldosteronism was negative but was positive for Cushing's syndrome (Table $1)$.

Surgery team evaluated the patient and recommended right adrenalectomy, which was the larger of the two adenomas. However, to assess if one of the adrenal nodule was producing excessive cortisol, adrenal venous sampling (AVS) was performed using dexamethasone $2 \mathrm{mg}$ oral every $6 \mathrm{~h}$ for $24 \mathrm{~h}$ 


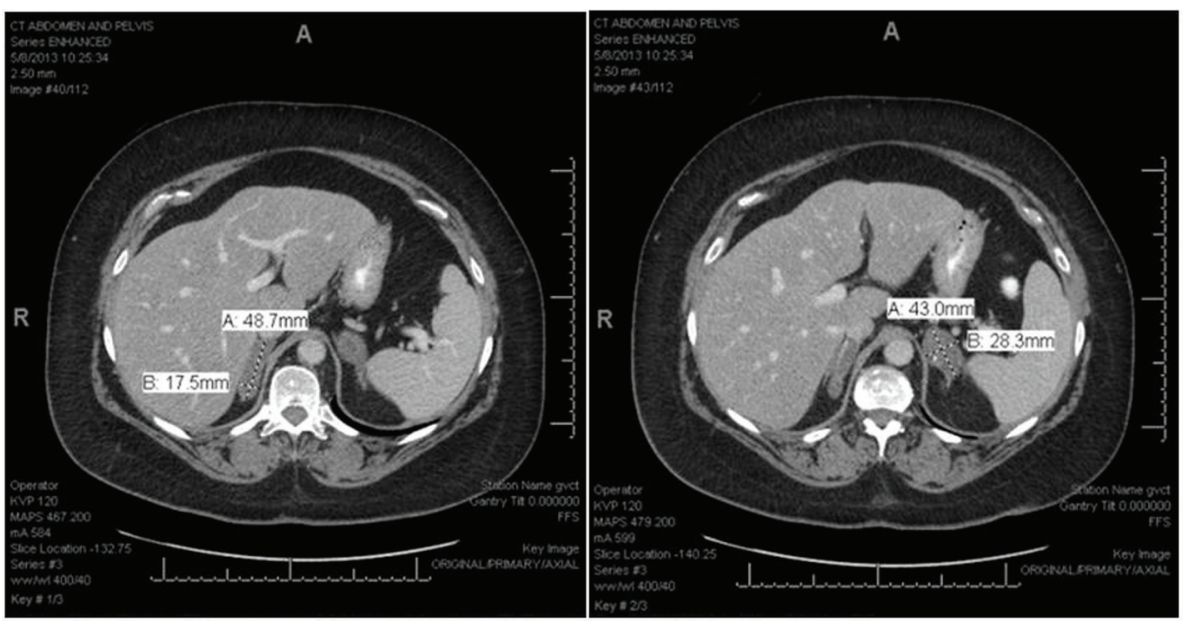

Figure 1. Right adrenal adenoma measuring $17.5 \times 48.7 \mathrm{~mm}$ and left adrenal adenoma with focus of calcification measuring 43 $\times 28.3 \mathrm{~mm}$.

preceding the procedure (Table 2).

Difference in adrenal gland epinephrine levels compared to peripheral levels was $>100 \mathrm{pg} / \mathrm{mL}$, suggesting successful localization of the catheter. Adrenal vein to peripheral vein cortisol ratio $>4$ was consistent with cortisol hypersecretion. Ratio of left to right adrenal cortisol levels $<2$ was diagnostic of bilateral cortisol hypersecretion [1]. It was determined patient should undergo right adrenalectomy as it was larger in size compared to the left adrenal adenoma.

The procedure was deferred as patient needed carotid artery surgery initially but the adrenal surgery was deferred since the patient had multiple co-morbidities directly resulting from hypercortisolism. She had several co-morbidities including uncontrolled hypertension on metoprolol and lisinopril, hypokalemia on potassium supplements, chronic leg cellulitis despite being on multiple antiobiotic courses for 10 months, glucose intolerance, and poor functional status requiring walker and cane. Ketoconazole was contraindicated for interactions with plavix and atorvastatin which could not discontinue due to her severe vascular disease. Mifepristone was initiated at $300 \mathrm{mg}$ PO every other day and titrated up to once daily. She had $65 \mathrm{lbs}(240 \mathrm{lbs}$ to $175 \mathrm{lb})$ weight loss, hypertension improved, had complete healing of cellulitis, and could ambulate independently by 4 months of starting mifepristone. Her potassium, liver function tests and BP were monitored closely.
Patient stopped taking mifepristone one week prior to the right adrenalectomy. Perioperatively, she was placed on hydrocortisone and eventually tapered off. After her right adrenal surgery, she did not require hydrocortisone. Symptomatically she had increased energy level, nausea and weakness resolution. Pathology showed a right adrenal cortical adenoma measuring $6.5 \times 5.0 \times 1.7 \mathrm{~cm}$ larger than on the CT scan dimension containing multiple adrenocortical nodules without any evidence of mitosis, necrosis or vascular invasion and the immunostaining was negative for adrenal carcinoma. After the first surgery, the patient expectedly had persistent hypercortisolism and mifepristone was reinitiated. Seven months later the patient opted to go for second surgery after consideration of continued medical management with mifepristone. The surgical pathology revealed a left adrenal cortical adenoma measuring $4.5 \times$ $2.6 \times 2.3 \mathrm{~cm}$. Adrenal insufficiency following her left adrenal surgery is being managed by hydrocortisone.

\section{Discussion}

This case had unique diagnostic dilemmas, initially the patient's source of hypercortisolism needed to be determined. The right adrenal adenoma was larger than the left adrenal tumor but the cortisol levels in the left were higher during

Table 1. Adrenal Adenoma Functional Evaluation

\begin{tabular}{ll}
\hline Test (normal range) & Result \\
\hline 24-h urine free cortisol $(4-50 \mu \mathrm{g} / 24 \mathrm{~h})$ & 89.2 \\
$1 \mathrm{mg}$ ODST cortisol $(5-15 \mu \mathrm{g} / \mathrm{dL})$ & 15.7 \\
$8 \mathrm{mg}$ ODST cortisol $(5-15 \mu \mathrm{g} / \mathrm{dL})$ & 20.0 \\
$11 \mathrm{pm}$ salivary cortisol $\times 2(<100 \mathrm{ng} / \mathrm{dL})$ & 156 and 151 \\
Random cortisol $(3-22 \mu \mathrm{g} / \mathrm{dL})$ & 17.3 \\
Random ACTH $(6-50 \mathrm{pg} / \mathrm{mL})$ & Undetectable \\
CRH stimulation test & No cortisol or ACTH response \\
\hline
\end{tabular}


Table 2. Adrenal Venous Sampling Site

\begin{tabular}{lll}
\hline & Cortisol $(\mathbf{3}-\mathbf{2 2} \boldsymbol{\mu g} / \mathbf{d L})$ & Epinephrine $(<\mathbf{8 4} \mathbf{~ p g} / \mathbf{m L})$ \\
\hline Left adrenal gland & 84.9 & 433 \\
Right adrenal gland & 56.5 & 1,911 \\
Inferior vena cava & 11.7 & 28 \\
\hline
\end{tabular}

adrenal venous sampling. Aldosterone measurement in AVS, reportedly used in case reports, could have provided important information during diagnosis, given the subtly low right adrenal cortisol and low left adrenal epinephrine levels (likely from phrenic vein dilution) but based on size and hypercortisolism bilateral adrenal resection was indicated [2, 3]. Persistent hypercortisolism increases the risk of perioperative morbidity by increasing infectious complications and delayed tissue healing [4]. The options of outpatient management of hypercortisolism include ketoconazole, metyrapone and mifepristone. Ketoconazole could not be used due to drug interactions with atorvastatin, and metyrapone is not readily available. The patient needed medical management for hypercortisolism during the interim before adrenalectomy given her multiple co-morbidities. Mifepristone is a glucocorticoid receptor antagonist that affinity 18 times greater than that of endogenous cortisol and has been FDA approved for inoperable Cushing's syndrome [5]. The evaluation of therapeutic response to mifepristone is based on clinical evaluation and not laboratory measurements. Side effects include hypokalemia, worsening blood pressure and increase risk for adrenal insufficiency [5]. Mifepristone has been FDA approved and studied in the SEISMIC trial with positive clinical reports in managing specific signs and symptoms of Cushing's syndrome [6]. The drug was utilized in this case to antagonize the hypercortisolism, which facilitated healing of her chronic cellulitis, optimized her functional status, improved her blood pressure and most importantly reduced her weight dramatically, all of which contributed to perioperative risk reduction and facilitated expeditious recovery from hypercortisolism prior to surgery. Avoiding the effects of abrupt steroid withdrawal by using mifepristone could have also played an important role in her return to normal functional status immediately after surgery. Mifepristone could potentially be used as bridge therapy when surgery needs to be deferred. To our knowledge, this is the first published case report using mifepristone in this unique role. Also it would be interesting to explore potential role of mifepristone preoperative preparation in patients with pituitary Cushing's syndrome.

\section{Disclosure}

The authors have nothing to disclose.

\section{References}

1. Young WF, Jr., du Plessis H, Thompson GB, Grant CS, Farley DR, Richards ML, Erickson D, et al. The clinical conundrum of corticotropin-independent autonomous cortisol secretion in patients with bilateral adrenal masses. World J Surg. 2008;32(5):856-862.

2. Bittner JGt, Brunt LM. Evaluation and management of adrenal incidentaloma. J Surg Oncol. 2012;106(5):557564.

3. Martins RG, Agrawal R, Berney DM, Reznek R, Matson M, Grossman AB, Druce MR. Differential diagnosis of adrenocorticotropic hormone-independent Cushing syndrome: role of adrenal venous sampling. Endocr Pract. 2012;18(6):e153-157.

4. van Heerden JA, Young WF, Jr., Grant CS, Carpenter PC. Adrenal surgery for hypercortisolism--surgical aspects. Surgery. 1995;117(4):466-472.

5. Cohan P. Pasireotide and mifepristone: new options in the medical management of Cushing's disease. Endocr Pract. 2014;20(1):84-93.

6. Fleseriu M, Biller BM, Findling JW, Molitch ME, Schteingart DE, Gross C. Mifepristone, a glucocorticoid receptor antagonist, produces clinical and metabolic benefits in patients with Cushing's syndrome. J Clin Endocrinol Metab. 2012;97(6):2039-2049. 